\title{
Lead-free BFN ceramics doped by chromium, lithium or manganese ${ }^{\star}$
}

\author{
Dariusz Bochenek ${ }^{\mathrm{a}}$, Przemysław Niemiec, and Małgorzata Adamczyk \\ University of Silesia, Institute of Technology and Mechatronics, 12, Żytnia St., 41-200 Sosnowiec, Poland
}

Received 11 June 2015 / Received in final form 30 July 2015

Published online 26 October 2015

(C) The Author(s) 2015. This article is published with open access at Springerlink.com

\begin{abstract}
The material of the study was lead-free $\mathrm{BaFe}_{0.5} \mathrm{Nb}_{0.5} \mathrm{O}_{3}$ ceramics subject to modification. The base composition $\mathrm{BaFe}_{0.5} \mathrm{Nb}_{0.5} \mathrm{O}_{3}$ as well as the chromium, lithium and manganese modified ones were obtained using conventional mixed oxides and carbonates method. Synthesis was performed by the powder calcination method at high temperature $1250{ }^{\circ} \mathrm{C}$ for $4 \mathrm{~h}$, while the densification was carried out by free sintering method under conditions $1350{ }^{\circ} \mathrm{C} / 4 \mathrm{~h}$. The paper presents a complex study of admixtures influence on the crystal structure, microstructure and dielectric properties of the BFN type samples. The mentioned dopants chromium, lithium or manganese in the BFN-type ceramics among other caused the reduction of the electric permittivity maximum as well as significant decrease in value of dielectric loss.
\end{abstract}

\section{Introduction}

The $\mathrm{BaFe}_{1 / 2} \mathrm{Nb}_{1 / 2} \mathrm{O}_{3}$ ceramics (BFN) may be an alternative material to replace lead-based ceramics such as: $\mathrm{PbFe}_{1-x} \mathrm{Nb}_{x} \mathrm{O}_{3}$ (PFN), which, due to its excellent piezoelectric and electromechanical properties, have been widely used for various devices, especially piezoelectric actuators, transformers and transducers [1-5]. The BFN, just like PFN, ceramics belong to the family of multiferroic materials [6] possessing perovskite structure of the general formula $\mathrm{ABO}_{3}$, where barium ions enter in the $\mathrm{A}$ positions, while iron and niobium ions alternately occupy the B position. The discussed materials have good ferroelectric (high values of dielectric permittivity) and the ferromagnetic properties at low (negative) temperatures, what was widely described in papers $[7,8]$. The electrophysical properties of the BFN ceramics were the subject of the following works $[8,9]$.

It is commonly known, that in order to achieve the high densification and optimal electrophysical parameters, sintering temperatures used in the process of preparation are very high and the time of the process is very long, it is one of the disadvantages of this material. However the main disadvantage significantly limiting the application possibilities of BFN is high value of dielectric losses [10]. The literature describes various methods for lowering this value, among other creation the solid solution $\mathrm{BFN}$ and glass compositions such as $\mathrm{Bi}_{2} \mathrm{O}_{3}-\mathrm{SiO}_{2}[11,12]$ or $\mathrm{Bi}_{2} \mathrm{O}_{3}-$ $\mathrm{Li}_{2} \mathrm{CO}_{3}$ [13]. The other method is connected with the

\footnotetext{
* Contribution to the Topical Issue "Materials for Dielectric Applications", edited by Maciej Jaroszewski and Sabu Thomas.

a e-mail: dariusz.bochenek@us.edu.pl
}

modification of BFN ceramics by suitable substitution, for example lanthanum ions [14]. To achieve the small value of dielectric loss the dopant, to be substituted at $\mathrm{A}$ or $\mathrm{B}$ site in $\mathrm{ABO}_{3}$ unit cell, should be suitably chosen. In the present paper authors described the influence of chromium, lithium and manganese modifiers on reduction of the dielectric losses of the basic material.

\section{Experiment}

The base research material was $\mathrm{BaFe}_{0.5} \mathrm{Nb}_{0.5} \mathrm{O}_{3}$ (BFN) obtained from a mixture of simple compounds $\mathrm{BaCO}_{3}$ (99.99\%), $\mathrm{Fe}_{2} \mathrm{O}_{3}(>99 \%)$ and $\mathrm{Nb}_{2} \mathrm{O}_{5}(99.99 \%)$. The $\mathrm{BFN}$ material was subjected to modification the following admixtures chromium, lithium or manganese in an amount of 1.0 at.\%. The mixture of the simple compositions was milled by Fritsch-type planetary ball mill for $15 \mathrm{~h}$ (wet in ethanol). As a result of doping of the three groups of compositions powders BFN type: chromium-doped (BFN-Cr), lithium-doped (BFN-Li) and doped with manganese (BFN-Mn) were obtained. Mixtures of the powders were synthesized by calcination method at temperature $1250{ }^{\circ} \mathrm{C}$ for $4 \mathrm{~h}$ at a linear heating rate of $300{ }^{\circ} \mathrm{C} / \mathrm{h}$. Compaction was carried out by the free sintering method at high temperature $\left(T_{s}=1350{ }^{\circ} \mathrm{C}\right.$ ) for $4 \mathrm{~h}$ (at a heating rate of $300{ }^{\circ} \mathrm{C} / \mathrm{h}$ ). For electrical tests on the surfaces of the samples were applied silver electrodes.

The X-ray tests were performed on a diffractometer Philips (with a $\mathrm{Cu}$ lamp and graphite monochromator) in the angular 2 from $12^{\circ}$ to $70^{\circ}$ (step $0.02^{\circ}$ and measurement time $4 \mathrm{~s} / \mathrm{step}$ ). The SEM images and the EDS (Energy Dispersive Spectrometry) were carried out using a scanning microscope HITACHI S-4700 with EDS Noran 


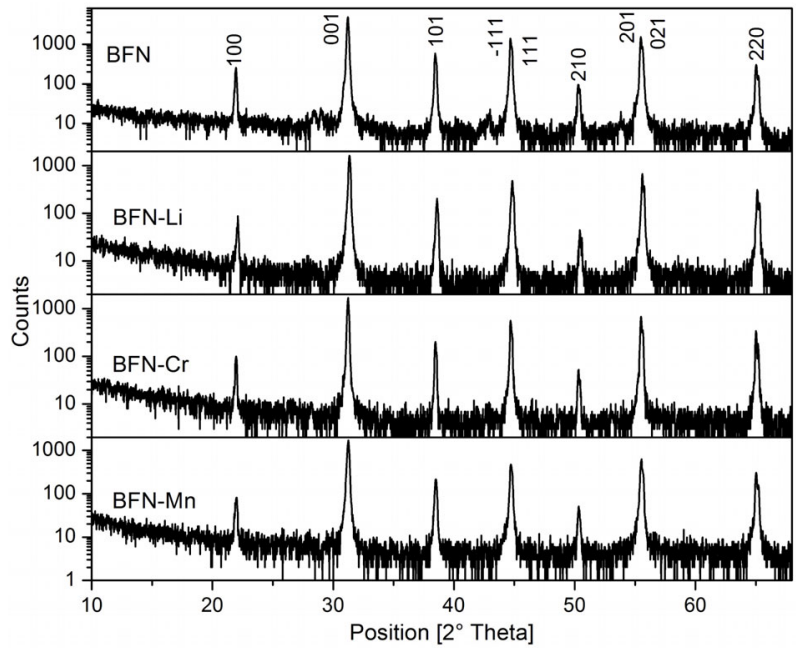

Fig. 1. X-ray diffraction patterns for BFN, BFN-Li, BFN-Cr and BFN-Mn powders.

Vantage. For testing of microstructure surfaces of the samples BFN type ceramics sputtered carbon. Temperature measurements of dielectric parameters were carried out on the QuadTech 1920 LCR meter (at frequencies of the measurement field from $0.02 \mathrm{kHz}$ to $1.0 \mathrm{MHz}$ ) in temperature range from $20^{\circ} \mathrm{C}$ to $380^{\circ} \mathrm{C}$ (the speed of temperature rise $2{ }^{\circ} \mathrm{C} / \mathrm{min}$, heating cycle). The $\mathrm{DC}$ conductivity measurements were investigated in temperature range from $20^{\circ} \mathrm{C}$ to $400{ }^{\circ} \mathrm{C}$ (heating cycle) using a $6517 \mathrm{~B}$ Keithley high resistance meter (voltage applied to the sample $100 \mathrm{~V}$ ).

\section{Results and discussions}

The X-ray diffraction patterns (in logarithmic scale) for all investigated compositions are presented in Figure 1. The $\mathrm{X}$-ray investigation shows that the pure BFN ceramicstype materials have the monoclinic symmetry with following cell parameters: $a=4.0743 \AA, b=4.0388 \AA$, $c=2.8759 \AA$ (the best fit was obtained for the pattern ICSD No. 00-057-0771 [15]). On the diffraction pattern are visible only the lines connected with the perovskite structure without any detectable lines of secondary phase. The introduction of chromium, lithium or manganese (in an amount of 1.0 at.\%) modifiers to the base BFN composition does not cause formation of additional (foreign) phases, so the doped materials at room temperature have the same monoclinic structure, as BFN ceramics. The real density was determined by measuring the mass and volume of the specimens. The additives caused to reduction of the density, which is as follows: $6.17 \mathrm{~g} / \mathrm{cm}^{3}$ (for BFN$\mathrm{Cr}$ ), $6.13 \mathrm{~g} / \mathrm{cm}^{3}$ (for BFN-Li), $6.07 \mathrm{~g} / \mathrm{cm}^{3}$ (for BFN-Mn) while the theoretical density is $6.54 \mathrm{~g} / \mathrm{cm}^{3}$ (reported in ICSD) [16]. Such reduction is connected with the increasing porosity of the modified BFN ceramics.

The microstructure of the undoped BFN ceramics is characterized by well developed, uniformed grain structure devoid of pore regions presence, as well as consisting of randomly oriented equiaxial grains (Fig. 2a). The microstructure of fracture BFN-Mn and BFN-Cr ceramics (Figs. 2d and 2c) looks similar to the microstructure of the BFN ceramics - has massed, densely packed, however significantly smaller grains. In case of ceramics modified by lithium the microstructure consists with much smaller, well-crystallized grains, the participation of pores significantly increased. It should be emphasized that grains of ceramics with additions of $\mathrm{Li}$ and $\mathrm{Cr}$ characterized by great uniformity of size, what influence on dielectric properties - especially the dielectric loss.

The EDS analysis was carried out on the ten randomly selected areas of the fracture surface of all discussed samples. The results of EDS analysis (Fig. 3) are in accordance with folded proportions of the starting components, calculated on a stoichiometric material in the preparation of the BFN-type ceramics.

Doping with $\mathrm{Li}, \mathrm{Mn}$ or $\mathrm{Cr}$ the BFN ceramics caused slightly decrease of the DC electrical conductivity (Fig. 4). The results of measurements show, that the lithium ions can replace basic cations (e.g. Fe) or they can occupy the interstitial positions in the crystal lattice. The variety of defects appearing in material examined as result of nonstoichiometry or doping allows to treat it as hard doped and partly compensated semiconductor [17].

In the case of admixing the BFN with $\mathrm{Cr}^{3+}$ chromium ion can replace the $\mathrm{Nb}^{5+}$ niobium ion in sublattice $\mathrm{B}$ $\left(W_{\mathrm{DB}}<W_{\mathrm{B}}\right)$ as a result of heterovalence admixing. The doping of this type cause the formation of additional holes, which may cause change the electric conductivity in BFN type ceramics. The $\mathrm{Cr}^{3+}$ chromium admixture may be also substituted by the $\mathrm{Fe}^{3+}$ iron ion as a result of isovalence admixing $\left(W_{\mathrm{DB}}=W_{\mathrm{B}}\right)$. In perovskite-like materials due to the small ionic radius excess of the chromium dopant can locate in the interstitial sites [17].

In the case of admixing the $\mathrm{BFN}$ with manganese $\mathrm{Mn}^{4+}$, (ion radius $52 \mathrm{pm}$ ) into account, can be substitute in positions $\mathrm{B}$ of the iron (67 pm) and niobium (69 pm) compound as a result of heterovalence admixing. In the first case admixing $\left(W_{\mathrm{DB}}>W_{\mathrm{B}}\right)$ causes the formation of additional holes. Such a type of admixing causes compensation of electric charges what leads to a decrease in electrical conduction of the BFN type ceramics. In the second case substitution of the $\mathrm{Mn}^{4+}$ ions in the $\mathrm{Nb}^{5+}$ niobium positions $\left(W_{\mathrm{DB}}<W_{\mathrm{B}}\right)$ introduces additional electrons, increasing electric conductivity.

The values of activation energy were calculated according to Arrhenius' law [18] and they are inside Figure 4.

Measurements of temperature dependences of dielectric permittivity reveal presence the anomalies (maximum), which is connected with the transition from ferroelectric to paraelectric phase (Fig. 5a). The maximum has broadened character, especially for BFN materials doped by lithium.

Each of the discussed compositions exhibits different properties in the phase transition regions, but in general it can be said that the chosen modifiers causes the shift of phase transition temperature to high values and decreases of maximum value of dielectric permittivity. High 

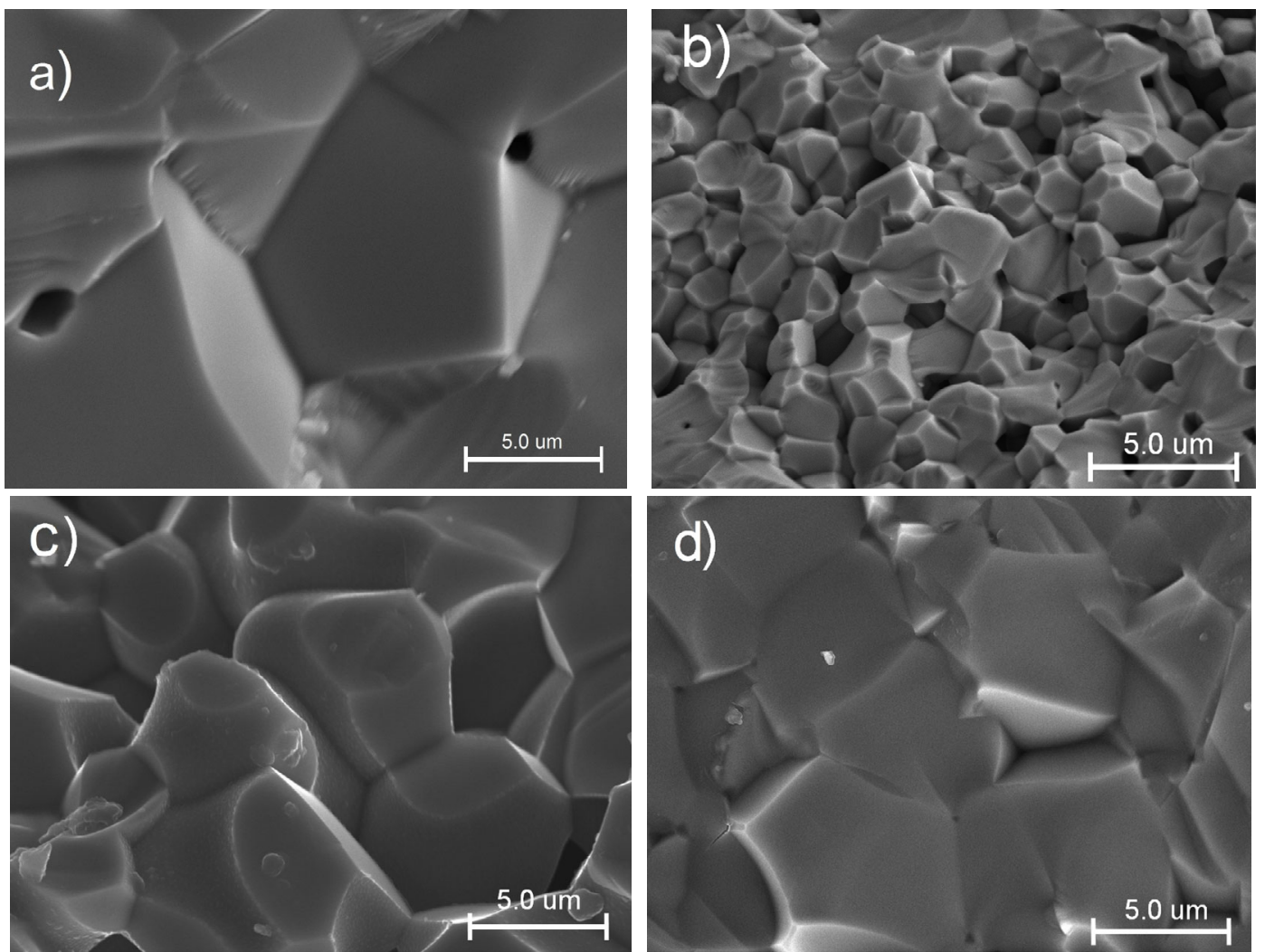

Fig. 2. SEM images of the microstructure of samples fractures: (a) BFN, (b) BFN-Li, (c) BFN-Cr, (d) BFN-Mn.



Fig. 3. EDS analysis of the element distribution for the BFN ceramics doped by: Cr (BFN-Cr), Li (BFN-Li) and Mn (BFN-Mn).

values of dielectric permittivity in undoped BFN ceramics occurring due to the possibility of the formation in the area with the electrode layers connector (metal - semiconductor) and the total capacity of the sample is the sum of the capacity of the base material and the resulting capacity those created layers [7]. The introduction of lithium and chromium ions disrupts layer system causes

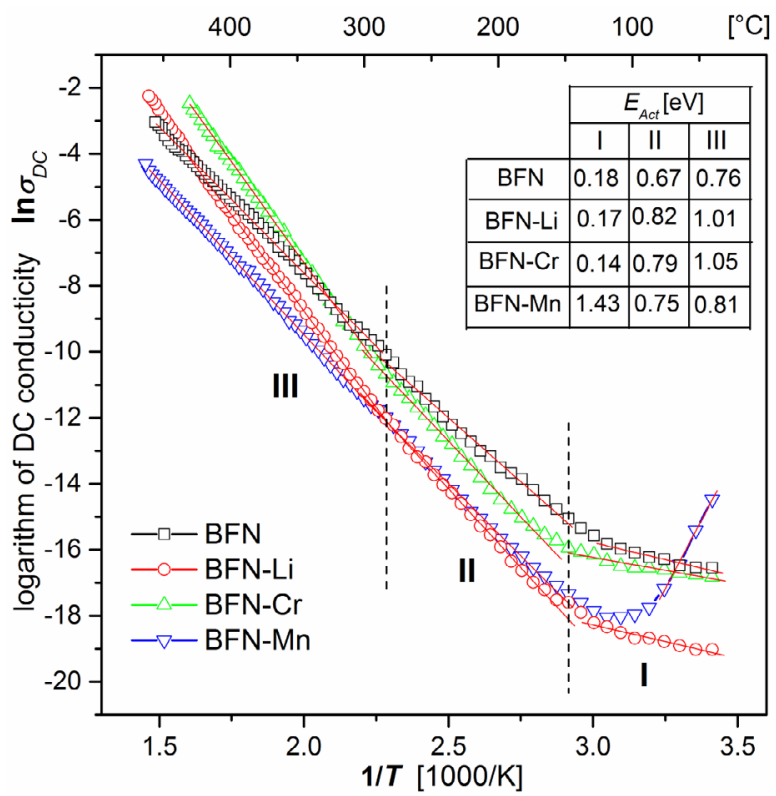

Fig. 4. An influence of the doped on the $\ln \sigma_{D C}(1 / T)$ relationship for the BFN ceramics.

a significant increase in the permittivity in the vicinity of room temperature, while decreasing its value at the phase transition. The manganium modification caused decreasing dielectric permittivity value in all investigated range of temperatures. 

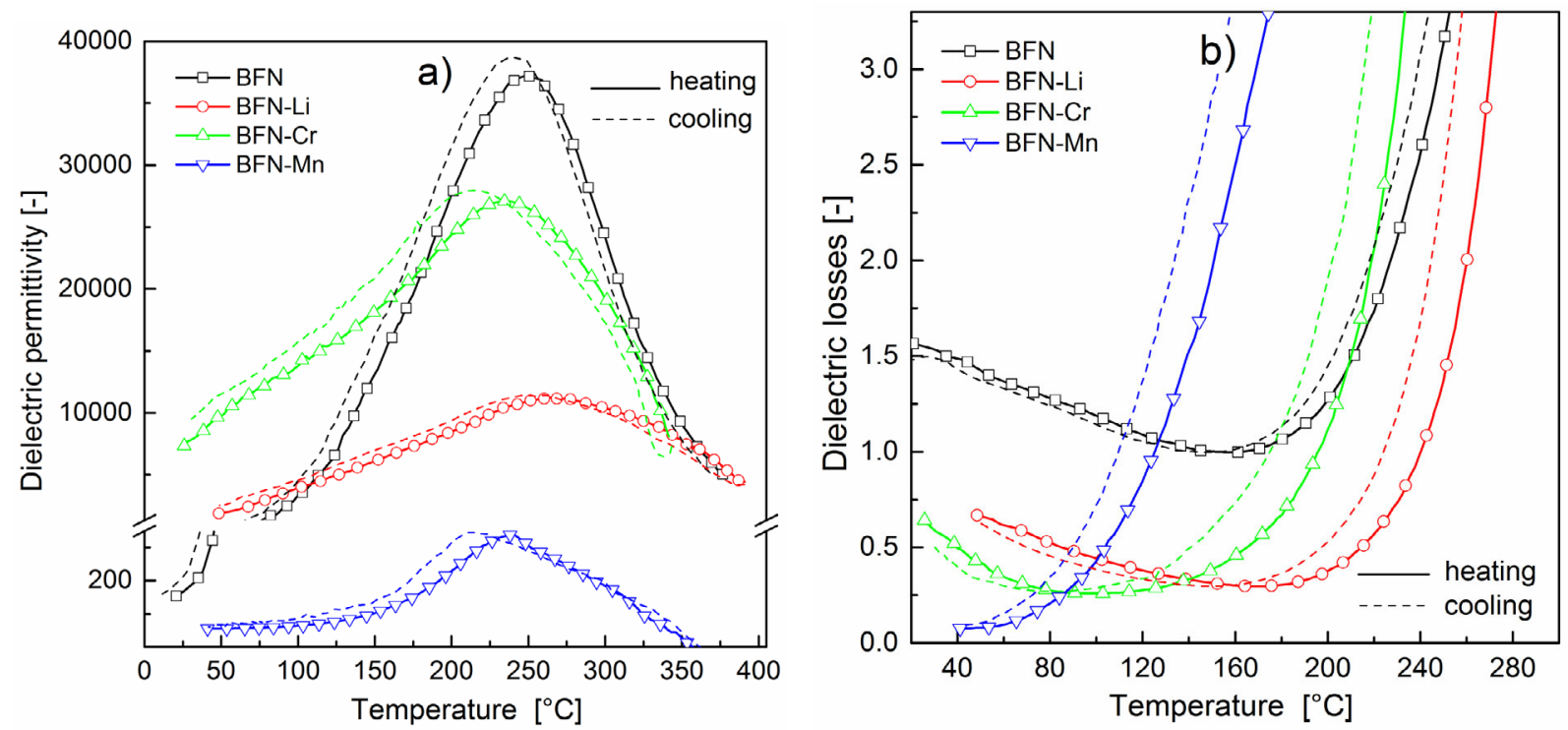

Fig. 5. Influence of the admixtures chromium, lithium and manganese for dielectric properties BFN ceramics.
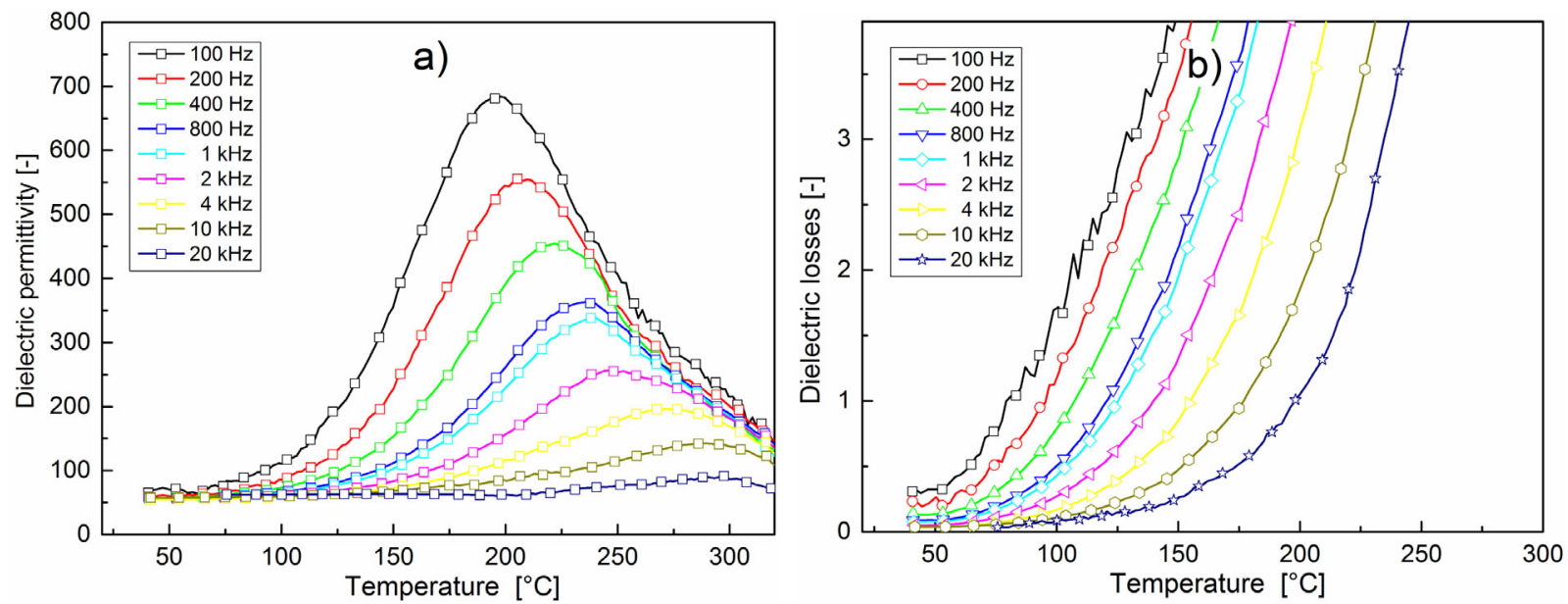

Fig. 6. Temperature dependences of dielectric properties for BFN ceramics doped by manganese: (a) $\varepsilon(T),(\mathrm{b}) \tan \delta(T)$.

All modifiers caused the significant reduction of dielectric losses (Fig. 5b). The BFN-Mn ceramics has low dielectric losses at room temperature (Fig. 6b) as compared to the compositions BFN-Li (Fig. 7b), BFN-Cr (Fig. 8b) and the pure BFN ceramics. The reduction is probably connected with the greater uniformity of grain size of admixtured ceramic materials. At higher temperatures (above $80{ }^{\circ} \mathrm{C}$ temperature) dielectric losses grow rapidly. In case of the $\mathrm{BFN}-\mathrm{Cr}$, dielectric losses remain at a low level to about $160^{\circ} \mathrm{C}$, whereas in the case of BFN-Li to about $200{ }^{\circ} \mathrm{C}$. Above these temperatures, occurs their rapid growth. The sharp growth of the dielectric losses at high temperatures is associated with the increase of electrical conductivity at high temperatures.

Moreover the modifiers caused the decrease in the diffuseness of phase transition. Figures 6-8 show the temperature dependence of the real part of dielectric permittivity and loss tangent of lithium, chromium and manganese modified BFN ceramics measured under various measuring frequencies. The temperature positions of dielectric permittivity maximum is strongly dependent on frequency of measuring field, i.e. maximum shifts to higher temperatures with increasing frequencies (Tab. 1). The greatest dispersion shows the BFN-Mn ceramics (in the temperature range $\sim 103{ }^{\circ} \mathrm{C}$ ), while the smallest shows the BFN-Li ceramics (in the temperature range $\sim 74^{\circ} \mathrm{C}$ ). Such behaviour indicates at the relaxor-like properties.

In the BFN compositions doped with manganese (Fig. 6) and with lithium (Fig. 7) occurs reduction of the value of dielectric permittivity. The temperature plot $\varepsilon(T)$ of the BFN-Mn ceramics is similar to the undoped BFN ceramics (Fig. 5). However, admixture of manganese inserted into the basic BFN, much decreases of the value of dielectric permittivity. In the case BFN doping with chromium (Fig. 8a) appears increase values of the dielectric permittivity, and simultaneously reduces the dielectric losses (Fig. 8b). 

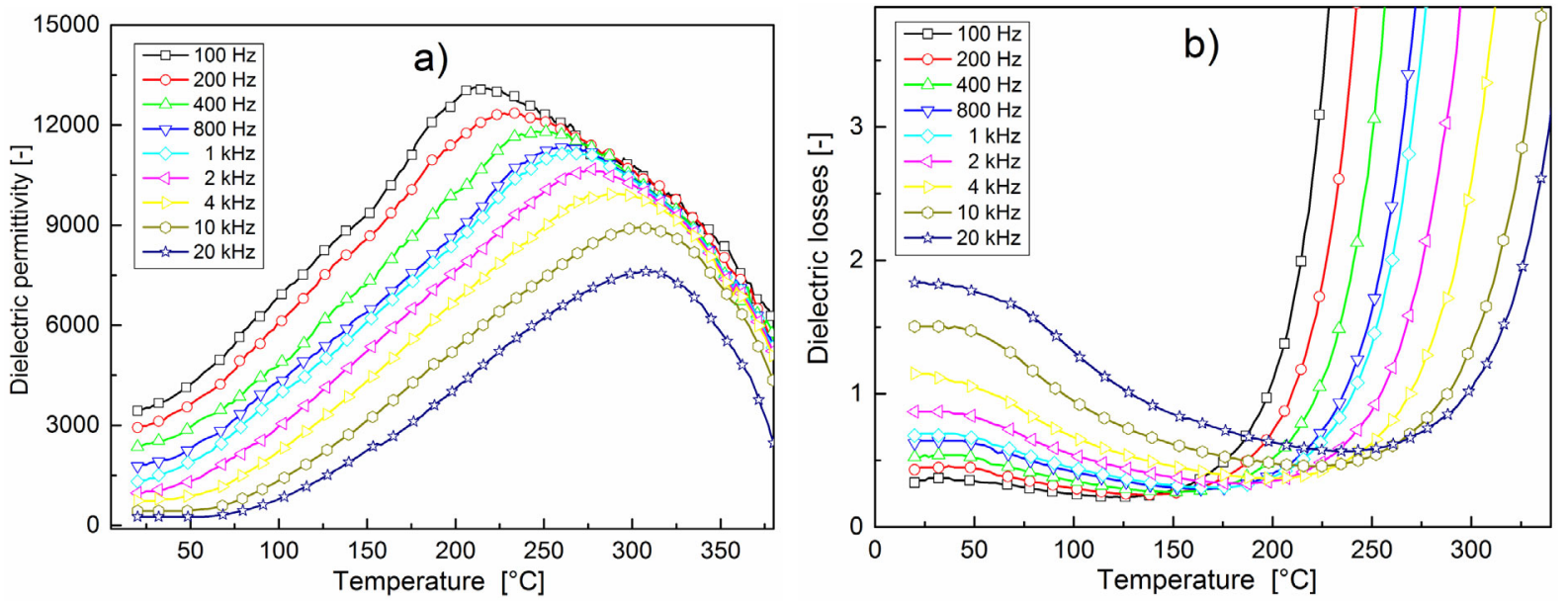

Fig. 7. Temperature dependences of dielectric properties for BFN ceramics doped by lithium: (a) $\varepsilon(T),(\mathrm{b}) \tan \delta(T)$.
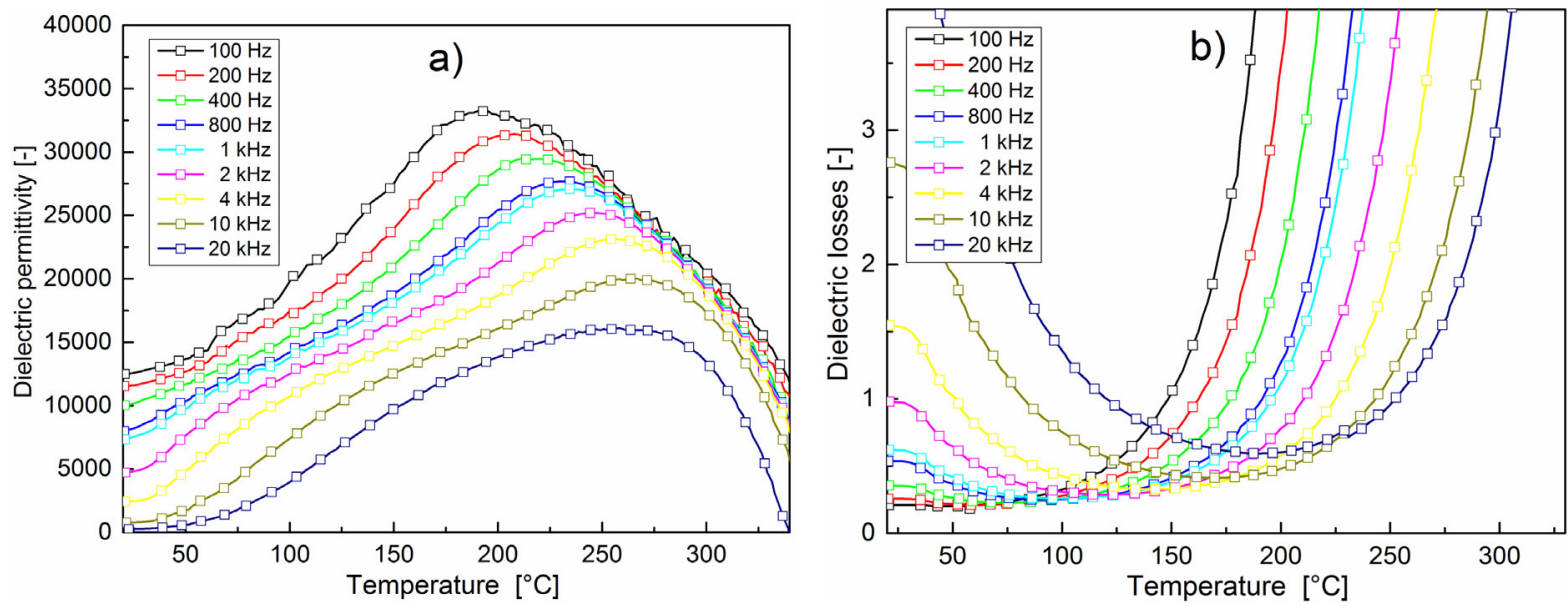

Fig. 8. Temperature dependences of dielectric properties for BFN ceramics doped by chromium: (a) $\varepsilon(T)$, (b) $\tan \delta(T)$.

Table 1. List of the temperatures (at $\varepsilon_{\max }$ ) for the measurement frequency for obtained samples.

\begin{tabular}{ccccc}
\hline & BFN & BFN-Cr & BFN-Li & BFN-Mn \\
\hline Frequency $[\mathrm{kHz}]$ & \multicolumn{4}{c}{$T_{m}\left[{ }^{\circ} \mathrm{C}\right]$} \\
\hline 0.1 & 186 & 216 & 192 & 196 \\
0.2 & 199 & 230 & 205 & 206 \\
0.4 & 209 & 249 & 218 & 220 \\
0.8 & 221 & 262 & 229 & 235 \\
1.0 & 225 & 264 & 234 & 237 \\
2.0 & 238 & 275 & 245 & 255 \\
4.0 & 250 & 291 & 256 & 271 \\
10.0 & 268 & 305 & 263 & 289 \\
20.0 & 276 & 307 & 266 & 299 \\
\hline
\end{tabular}

\section{Conclusion}

The lead-free $\mathrm{BaFe}_{0.5} \mathrm{Nb}_{0.5} \mathrm{O}_{3}$ ceramics may be an alternative material for leaded ceramics (e.g. PZT-type ceramics, PFN ceramics, etc.) widely used in many fields of microelectronic and micromechatronics. The ferroelectromagnetic BFN ceramics shows a behavior typical for relaxor materials with the characteristic occurrence of frequency dispersion. In addition to the high values of dielectric permittivity BFN ceramics has a high value of dielectric losses, which can be reduced by the introduction of the basic BFN composition of the suitable admixtures.

Compared with undoped BFN ceramics, doped ceramic compositions of the BFN type (doped by manganese, lithium and chromium amount 1.0 at.\%) have smaller dielectric losses as well as lower DC electrical conductivity, but also much smaller value of dielectric permittivity. Doped compositions of the BFN type also exhibit extension of the phase transition area.

Reduction of dielectric losses in doped BFN type ceramics significantly increases the application possibilities ceramics in many areas of modern microelectronics and micromechatronics.

\section{References}

1. Y. Yu, R.N. Singh, J. Appl. Phys. 88, 7249 (2000)

2. D. Bochenek, Z. Surowiak, J. Alloys Compd. 480, 732 (2009)

3. D. Bochenek, J. Dudek, Eur. Phys. J. Special Topics 154, $19(2008)$ 
4. D. Bochenek, Z. Surowiak, Phys. Status Solidi A 206, 2857 (2009)

5. S.B. Majumder, S. Bhattacharyya, R.S. Katiyar, A. Manivannan, P. Dutta, M.S. Seehra, J. Appl. Phys. 99, 024108 (2006)

6. H. Schmid, J. Phys.: Condens. Matter 20, 434201 (2008)

7. U. Intatha, S. Eitssayeam, J. Wang, T. Tunkasiri, Curr. Appl. Phys. 10, 21 (2010)

8. D. Bochenek, Z. Surowiak, J. Poltierova-Vejpravova, J. Alloys Compd. 487, 572 (2009)

9. P.K. Patel, J. Rani, N. Adhlakha, H. Singh, K.L. Yadav, J. Phys. Chem. Solids 4, 545 (2013)

10. S. Ke, H. Fan, H. Huang, J. Electroceram. 22, 252 (2009)

11. J.W. Zhai, X. Yao, X.O. Cheng, L.Y. Zhang, C. Haydn, J. Mater. Sci. 37, 3739 (2002)

12. P.V. Divya, V. Kumar, J. Am. Ceram. 90, 472 (2007)

13. T. Hu, H. Jantunen, A. Deleniv, S. Leppavuori, S. Gevorgian, J. Am. Ceram. 87, 578 (2004)
14. S. Kumar Kar, S. Swain, S. Pawan Kumar, Mater. Chem. Phys. 155, 171 (2015)

15. S. Saha, T.P. Sinha, J. Phys.: Condens. Matter 14, 249 (2002)

16. The Inorganic Crystal Structure database (ICSD), code 43622

17. K. Wójcik, K. Zieleniec, M. Milata, Ferroelectrics 289, 107 (2003)

18. D. Bochenek, P. Kruk, R. Skulski, P. Wawrzała, J. Electroceram. 26, 8 (2011)

Open Access This is an open access article distributed under the terms of the Creative Commons Attribution License (http://creativecommons.org/licenses/by/4.0), which permits unrestricted use, distribution, and reproduction in any medium, provided the original work is properly cited. 